Algebraic $8 \mathcal{G}$ Geometric $\mathcal{T}$ opology

Volume 4 (2004) 49-71

Published: 7 February 2004

ATG

\title{
The boundary-Wecken classification of surfaces
}

\author{
Robert F. BRown \\ Michael R. Kelly
}

\begin{abstract}
Let $X$ be a compact 2-manifold with nonempty boundary $\partial X$ and let $f:(X, \partial X) \rightarrow(X, \partial X)$ be a boundary-preserving map. Denote by $M F_{\partial}[f]$ the minimum number of fixed point among all boundary-preserving maps that are homotopic through boundary-preserving maps to $f$. The relative Nielsen number $N_{\partial}(f)$ is the sum of the number of essential fixed point classes of the restriction $\bar{f}: \partial X \rightarrow \partial X$ and the number of essential fixed point classes of $f$ that do not contain essential fixed point classes of $\bar{f}$. We prove that if $X$ is the Möbius band with one (open) disc removed, then $M F_{\partial}[f]-N_{\partial}(f) \leq 1$ for all maps $f:(X, \partial X) \rightarrow(X, \partial X)$. This result is the final step in the boundary-Wecken classification of surfaces, which is as follows. If $X$ is the disc, annulus or Möbius band, then $X$ is boundary-Wecken, that is, $M F_{\partial}[f]=N_{\partial}(f)$ for all boundary-preserving maps. If $X$ is the disc with two discs removed or the Möbius band with one disc removed, then $X$ is not boundary-Wecken, but $M F_{\partial}[f]-N_{\partial}(f) \leq$ 1. All other surfaces are totally non-boundary-Wecken, that is, given an integer $k \geq 1$, there is a map $f_{k}:(X, \partial X) \rightarrow(X, \partial X)$ such that $M F_{\partial}\left[f_{k}\right]-$ $N_{\partial}\left(f_{k}\right) \geq k$.
\end{abstract}

AMS Classification 55M20; 54H25, 57N05

Keywords Boundary-Wecken, relative Nielsen number, punctured Möbius band, boundary-preserving map

\section{Introduction}

Given a map $f: X \rightarrow X$ of a compact $n$-manifold with (possibly empty) boundary to itself, let $N(f)$ denote the Nielsen number of $f$, that is, the number of essential fixed point classes. For $M F[f]$ the minimum number of fixed points among all maps homotopic to $f$, we have $N(f) \leq M F[f]$. Wecken proved in [15] that if $X$ is an $n$-manifold with $n \geq 3$, then $N(f)=M F[f]$ for all maps $f: X \rightarrow X$. Consequently, the property: $N(f)=M F[f]$ for every map $f: X \rightarrow X$ has come to be called the Wecken property of a manifold $X$ and 
a manifold with this property is said to be a Wecken manifold. If $X$ is a 2 manifold with Euler characteristic non-negative, then $X$ is a Wecken manifold. The Wecken property of the Klein bottle is a consequence of Theorem 5.1 of [4] (see Corollary 8.3 of [5]), the property is verified for the torus in [1] and for the projective plane in 8 . The verification of the Wecken property for the four remaining such 2-manifolds (sphere, disc, annulus, Möbius band) is easy.

On the other hand, if $X$ is a 2-manifold with negative Euler characteristic, then it is proved in [6] that $X$ is totally non-Wecken, that is, given an integer $k \geq 1$, there is a map $f_{k}: X \rightarrow X$ such that $M F\left[f_{k}\right]-N\left(f_{k}\right) \geq k$. Thus we have

Theorem 1 (Wecken classification of surfaces) Let $X$ be a compact 2-manifold.

(a) If the Euler characteristic of $X$ is non-negative, then $X$ is Wecken.

(b) All other surfaces are totally non-Wecken.

Now let $X$ denote a compact $n$-manifold with nonempty boundary $\partial X$ and consider boundary-preserving self-maps $f:(X, \partial X) \rightarrow(X, \partial X)$. We write the restriction of $f$ to the boundary as $\bar{f}: \partial X \rightarrow \partial X$. The minimum number of fixed points among all maps of pairs homotopic to $f$ through boundarypreserving maps is written as $M F_{\partial}[f]$. The relative Nielsen number, that we denote by $N_{\partial}(f)$, is defined to be the sum of the number of essential fixed point classes of $\bar{f}$ and the number of essential fixed point classes of $f$ that do not contain essential fixed point classes of $\bar{f}$. We always have $N_{\partial}(f) \leq M F_{\partial}[f]$ and the $n$-manifold $X$ is said to be boundary-Wecken if $N_{\partial}(f)=M F_{\partial}[f]$ for all maps of pairs $f:(X, \partial X) \rightarrow(X, \partial X)$. Schirmer 13 proved that all $n$-manifolds with nonempty boundary are boundary-Wecken if $n \geq 4$.

A compact $n$-manifold $X$ with nonempty boundary is said to be totally nonboundary-Wecken if, given an integer $k \geq 1$, there is a map $f_{k}:(X, \partial X) \rightarrow$ $(X, \partial X)$ such that $M F_{\partial}\left[f_{k}\right]-N_{\partial}\left(f_{k}\right) \geq k$. The manifold $X$ is said to be almost boundary-Wecken if $X$ is not boundary-Wecken but there exists an integer $B \geq 1$ such that $M F_{\partial}[f]-N_{\partial}(f) \leq B$ for all maps $f:(X, \partial X) \rightarrow(X, \partial X)$.

The purpose of this paper is to prove the following theorem:

Theorem 2 Let $X$ be the Möbius band with one (open) disc removed and let $f:(X, \partial X) \rightarrow(X, \partial X)$ be any map, then $M F_{\partial}[f]-N_{\partial}(f) \leq 1$.

This result will allow us to complete the proof of the following: 
Theorem 3 (Boundary-Wecken classification of surfaces) Let $X$ be a compact 2-manifold with nonempty boundary.

(a) If $X$ is the disc, annulus or Möbius band, then $X$ is boundary-Wecken.

(b) If $X$ is the disc with two discs removed or the Möbius band with one disc removed, then $X$ is almost boundary-Wecken, with $B=1$.

(c) All other surfaces with boundary are totally non-boundary-Wecken.

Proof Conclusion (a) is proved in [2]. With regard to conclusion (b), the proof that the disc with two discs removed is not boundary-Wecken is in [10] and it is proved in [2] that it is almost boundary-Wecken with $B=1$. It is shown in 11] that the Möbius band with one disc removed is not boundary-Wecken so Theorem 2 will complete the proof of conclusion (b). The proof that the disc with three or more discs removed is totally non-boundary-Wecken is in 12. For the Möbius band with two or more discs removed, the proof that it is totally non-boundary-Wecken is in [11. For $X$ any surface not yet listed, results from [2] and [10] imply that $X$ is totally non-boundary-Wecken. Thus we have conclusion (c).

To begin the proof of Theorem 2, we show in Section 1 that, for $X$ the Möbius band with one disc removed, any map $f:(X, \partial X) \rightarrow(X, \partial X)$ can be homotoped as a map of pairs to a convenient "reduced form". For a map in that form, the inverse images of the arcs where handles are attached to a disc to form $X$ are properly embedded 1-dimensional submanifolds of $X$. We classify the possible such submanifolds in Section 1 also.

Now let $C_{1}$ and $C_{2}$ be the components of $\partial X$ and let $f_{i}: C_{i} \rightarrow \partial X$ be the restriction of $f$ to $C_{i}$ for $i=1,2$. The following terminology will be convenient for presenting the various cases to be checked in order to prove Theorem 2. If both the $f_{i}$ are essential maps, we will say that $f$ is boundary essential. If both $f_{i}$ are inessential maps, then $f$ is boundary inessential. If one of the maps $f_{1}$ or $f_{2}$ is essential and the other is inessential, we say that $f$ is boundary semiessential. Also, as in [2], we let $\operatorname{Im}_{\partial}(f)$ denote the number of components of $\partial X$ that contain points of $f(\partial X)$.

In Section 2, we prove Theorem 2 in the case that $f$ is boundary inessential. Section 3 is devoted to the proof of Theorem 2 when $f$ is boundary essential. We complete the proof of Theorem 2 in Section 4, by proving it for boundary semi-essential maps.

Brian Sanderson helped us with the proof of Therem 8. We also thank the referee who gave us many constructive suggestions that have significantly improved the exposition. 


\section{Reduced form}

Notation In Figure 1, we represent the punctured Möbius band $X$ as a union of discs $X=D \cup H_{1} \cup H_{2}$ where the handle $H_{1}$ is twisted, that is, $D \cup H_{1}$ is a Möbius band, and the handle $H_{2}$ is not twisted: $D \cup H_{2}$ is an annulus. We have $D \cap H_{1}=A_{1} \cup A_{2}$ and $D \cap H_{2}=A_{3} \cup A_{4}$ and we let $A$ be the union of all the $\operatorname{arcs} A_{j}$. We denote the arc that is the intersection of $\partial D$ with the component $C_{2}$ of $\partial X$ by $T_{2}$ and the three arcs that are the components of $\partial D \cap C_{1}$ are called $T_{1}, T_{3}$ and $T_{4}$, as the figure shows. We choose points $x_{1} \in T_{1}$ and $x_{2} \in T_{2}$ as well as $x_{0} \in$ int $X$ on the line segment, that we denote by $\left[x_{1}, x_{2}\right]$, with endpoints $x_{1}$ and $x_{2}$. The simple closed curves $h_{1}$ and $h_{2}$ generate the fundamental group of $X$ based at $x_{0}$.

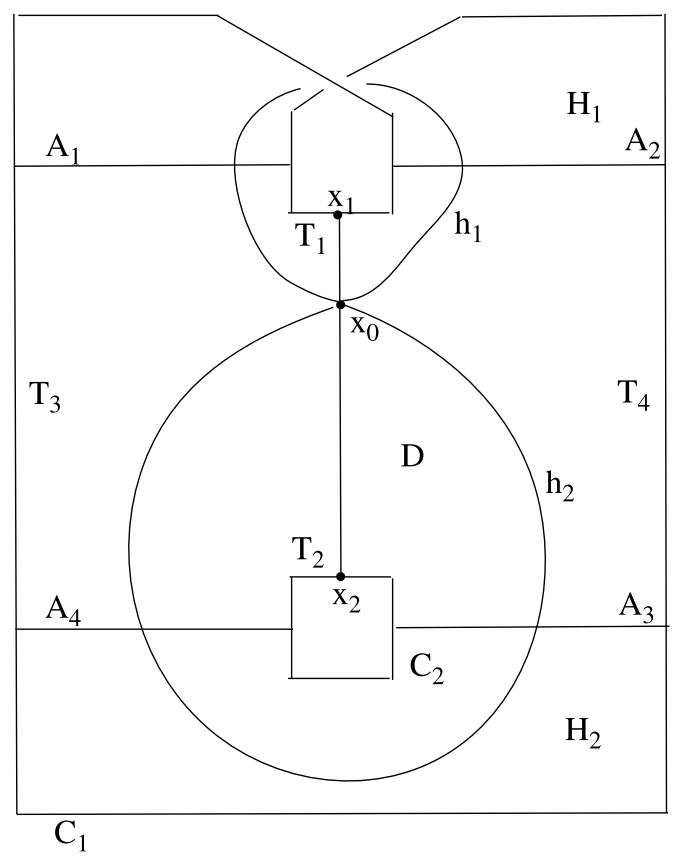

Figure 1

For a map $f:(X, \partial X) \rightarrow(X, \partial X)$, the restriction of $f$ to the boundary component $C_{i}$ will always be called $f_{i}$ and we will use $C_{i \#}$ to denote the component of $\partial X$ containing $f\left(C_{i}\right)$. Thus, corresponding to the map $f$, for $i=1,2$ we have the maps of simple closed curves $f_{i}: C_{i} \rightarrow C_{i \#}$. The absolute value of the degree of a map between simple closed curves is independent of the orientations of the curves, so it can be used without specifying orientations. For that reason, 
by the degree of a map between simple closed curves we will mean the absolute value. In particular, we denote the degrees of the maps $f_{i}$ by $\left|d_{i}\right|$.

In order to analyze the boundary-preserving maps of $X$, we will need the classification of the simple closed curves in the interior of $X$ that are transverse to $A$ and have minimal intersection with it, up to isotopy. That is the purpose of the first two lemmas.

Lemma 1 Let $K$ be a simple closed curve in the interior of $X$ that is transverse to $A$ and such that the number of points in $K \cap A$ is minimal within the isotopy class of $K$. Then each of $K \cap H_{1}$ and $K \cap H_{2}$ has at most two components.

Proof Write $K \cap H_{1}$ as a union of its components as $K \cap H_{1}=J_{1} \cup \ldots \cup$ $J_{r_{1}}$. Each component $J_{j}$ is an arc with its endpoints in $A$. If there is any components of $K \cap H_{1}$ with both endpoints in the same $A_{i}$, take the innermost such component, then it bounds a disc in $H_{1}-K$ that can be used to reduce $K \cap A$ by two points, contrary to the assumption of minimal intersection. Thus we may assume that each component $J_{j}$ has endpoints $p_{1 j} \in A_{1}$ and $p_{2 j} \in A_{2}$. Orienting the boundary of $D$ in a clockwise direction induces an ordering on the $A_{i}$. Ordering the $J_{j}$ so that $p_{1 j}<p_{1, j+1}$ in the ordering of $A_{1}$ then, since the handle $H_{1}$ is twisted, $p_{2 j}<p_{2, j+1}$ for all $j$. Let $L_{1} \subset K \cap\left(D \cup H_{2}\right)$ be the component containing $p_{11}$ and let $x$ be the other endpoint of $L_{1}$. If $x=p_{21}$, then $J_{1} \cup L_{1}$ is a simple closed curve so $r_{1}=1$. Now suppose $x \neq p_{21}$. If $x \neq p_{2 r_{1}}$ then $p_{2 r_{1}}$ and the subarc of $A_{1}$ between $p_{11}$ and $p_{1 r_{1}}$ are in different components of $\left(D \cup H_{2}\right)-L_{1}$ (which is disconnected because both endpoints of the arc $L_{1}$ are in the same component of the boundary of the annulus $D \cup H_{2}$ ). Since there could not then be an component of $K \cap\left(D \cup H_{2}\right)$ with endpoint $p_{2 r_{1}}$, we conclude that the endpoints of $L_{1}$ are $p_{11}$ and $p_{2 r_{1}}$. A symmetric argument shows that if $L_{2}$ is the component of $K \cap\left(D \cup H_{2}\right)$ containing $p_{21}$ then the other endpoint is $p_{1 r_{1}}$. Since $J_{1} \cup L_{1} \cup J_{2} \cup L_{2}$ is a simple closed curve, we conclude that $r_{1} \leq 2$.

Writing $K \cap H_{2}=J_{1}^{\prime} \cup \ldots \cup J_{r_{2}}^{\prime}$, the arcs $J_{j}^{\prime}$ have endpoints $p_{3 j} \in A_{3}$ and $p_{4 j} \in A_{4}$. Ordering the $J_{j}^{\prime}$ so that $p_{3 j}<p_{3, j+1}$ in the ordering of $A_{3}$ induced by the orientation of the boundary of $D$ then, since the handle $H_{2}$ is not twisted, $p_{4 j}>p_{4, j+1}$ for all $j$. Letting $L_{1}^{\prime}$ denote the component of $K \cap\left(D \cup H_{1}\right)$ containing $p_{31}$, if the other endpoint is $p_{41}$, then $J_{1}^{\prime} \cup L_{1}^{\prime}$ is a simple closed curve and $r_{2}=1$. Thus we suppose that endpoint is not $p_{41}$. If $L_{1}^{\prime} \subset D$, then $p_{41}$ and the subarc of $A_{3}$ between $p_{31}$ and $p_{3 r_{2}}$ are in different components of $D-L_{1}^{\prime}$. Since there could not then be a component of $K \cap\left(D \cup H_{1}\right)$ with 
endpoint $p_{41}$, we conclude that $L_{1}^{\prime} \cap H_{1}$ is an arc. A symmetric argument shows that if $L_{r_{2}}^{\prime} \subset D$ then no component of $K \cap\left(D \cup H_{1}\right)$ could have endpoint $p_{4 r_{2}}$ and so $L_{r_{2}}^{\prime} \cap H_{1}$ is also an arc. Since we showed in the first part of the proof that there are at most two components of $K \cap H_{1}$, we conclude that $r_{2} \leq 2$.

Let $K$ be a simple closed curve in the interior of $X$ that is transverse to $A$. If $K \cap H_{j}$ has $r_{j}$ components for $j=1,2$, then we will say that $K$ is of type $\left(r_{1}, r_{2}\right)$. A simple closed curve in $X$ is called inessential if it bounds a disc and essential otherwise.

Lemma 2 Let $K$ be an essential simple closed curve in the interior of $X$ that is transverse to $A$ and such that the number of points in $K \cap A$ is minimal within the isotopy class of $K$. Then the type $\left(r_{1}, r_{2}\right)$ of $K$ is one of the following: $(1,0),(2,0),(0,1),(1,1),(2,1)$ or $(2,2)$,

Proof By Lemma 1, we know that the possible values of the $r_{j}$ are 0,1 and 2 . If $\left(r_{1}, r_{2}\right)=(0,0)$ then $K$ would bound a disc, in $D$, which is excluded since $K$ is essential. We claim that $K$ cannot be of type $(0,2)$ or $(1,2)$. We assume $r=2$ and we establish a contradiction as follows. Let $J_{1}^{\prime}$ be the component of $K$ in $H_{2}$ with endpoints $p_{j 1}$ for $j=3,4$ and let $L_{1}^{\prime}$ be the component in $D \cup H_{1}$ with endpoint $p_{41}$, then the other endpoint must be $p_{32}$ (because if it were $p_{31}$ then $J_{1}^{\prime} \cup L_{1}^{\prime}$ would be a simple closed curve and if it were $p_{42}$ that would contradict minimality because either $L_{1}^{\prime}$ or $L_{2}^{\prime}$ could be eliminated). If $L_{1}^{\prime} \subset D$, then $p_{31}$ and $p_{42}$ are in different components of $\left(D \cup H_{1}\right)-L_{1}^{\prime}$ and so the case $\left(r_{1}, r_{2}\right)=(0,2)$ cannot occur. If $L_{1}^{\prime}$ intersects $H_{1}$, then, since $p_{11}$ and $p_{21}$ are in different components of $D-L_{1}^{\prime}$, the case $\left(r_{1}, r_{2}\right)=(1,2)$ also cannot occur. The six cases remaining are shown in Figure 2.

We will now describe a convenient form for a map $f:(X, \partial X) \rightarrow(X, \partial X)$ and then prove that we can always obtain it through a boundary-preserving homotopy. An arc in $X$ is properly embedded if it intersects $\partial X$ in its endpoints and at no other point. A compact 1-dimensional submanifold of $X$ is said to be properly embedded if it is a union of simple closed curves in the interior of $X$ and properly embedded arcs. A properly embedded arc $K$ in $X$ is inessential if there is an arc $L$ in $\partial X$ connecting the endpoints of $K$ such that the simple closed curve $K \cup L$ bounds a disc in $X$. Otherwise, the properly embedded arc $K$ is essential.

Definition A map $f:(X, \partial X) \rightarrow(X, \partial X)$ is said to be in reduced form if it has the following properties: 


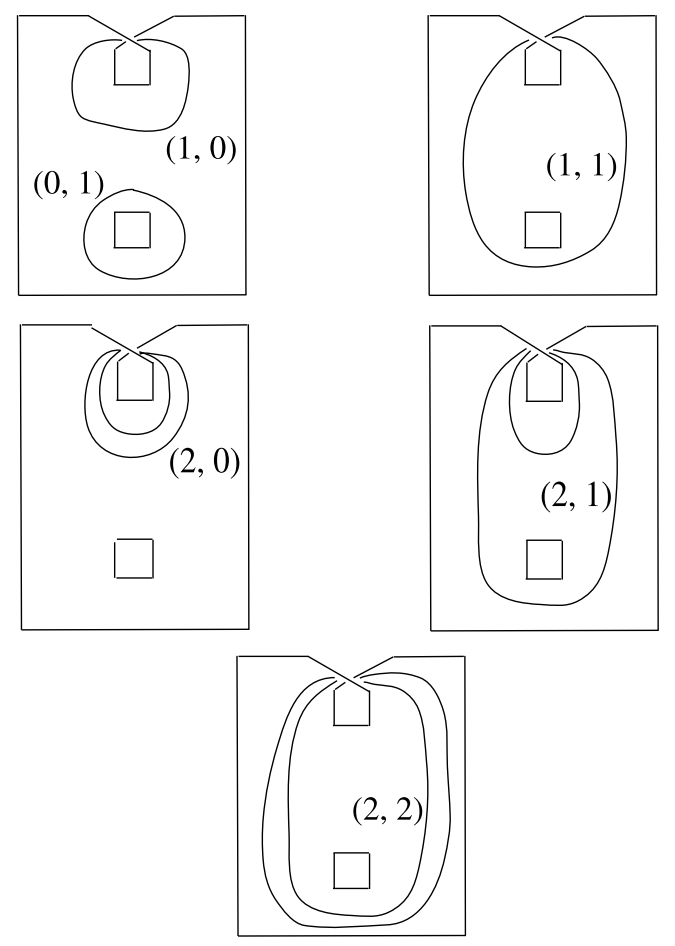

Figure 2

(1) for $i=1,2$, the map $f_{i}: C_{i} \rightarrow C_{i \#}$ is the constant map to $x_{i \#}$ if $\left|d_{i}\right|=0$ and a $\left|d_{i}\right|$-to-one map otherwise,

(2) $f^{-1}(A)$ is a properly embedded 1-dimensional submanifold transverse to $A$ such that each component of $f^{-1}(A)$ has minimal intersection with $A$ within its proper isotopy class,

(3) all the components of $f^{-1}(A)$ are essential and

(4) if $\lambda$ is an arc in $A$ with endpoints in $f^{-1}(A)$ and interior disjoint from $f^{-1}(A)$, then the endpoints of $\lambda$ are mapped to distinct components of $A$.

Lemma 3 Given a map $f:(X, \partial X) \rightarrow(X, \partial X)$, there is a map $g$ in reduced form that is homotopic, as a map of pairs, to $f$.

Proof The restriction of $f$ to $\partial X$ can be made to satisfy condition (1) by the homotopy extension theorem. Without changing the map on the boundary, and thus condition (1) is retained, the map can then be modified, using the 
techniques of [9], so that the inverse image of $A$ is a properly embedded 1submanifold whose components make minimal intersection, within their proper homotopy class, with $A$. In this way, (2) is also satisfied. For condition (3), components that are inessential simple closed curves can be eliminated by an "innermost simple closed curve" argument and, similarly, inessential properly embedded arcs can be eliminated by an "outermost arc" argument. To simplify the notation, let us suppose that $f$ already satisfies conditions (1), (2) and (3) of the definition of reduced form, then it remains to homotope $f$ so that, if $\lambda$ is an arc in $A$ with endpoints in $f^{-1}(A)$ and interior disjoint from $f^{-1}(A)$, then the endpoints of $\lambda$ are mapped to distinct components of $A$. To accomplish this, suppose the endpoints of such an arc $\lambda$ are mapped to the same component of $A$. Since the interior of $\lambda$ does not contain points of $f^{-1}(A)$, the path $f(\lambda)$ either lies entirely in $D$ or entirely in one of the handles $H_{j}$. If $f(\lambda)$ lies in $D$ then, since $f^{-1}(A)$ is transverse to $A$, there are neighborhoods of the endpoints of $\lambda$ in $A$ whose image lie in some handle $H_{j}$. We can homotope $f$ so that the image of a neighborhood of $\lambda$ in $A$ lies entirely in that handle. Similarly, if $f(\lambda)$ lies in some handle $H_{j}$, then we can homotope $f$ so that the neighborhood of $\lambda$ is mapped to $D$. Repeating this procedure a finite number of times we obtain a map in reduced form homotopic to $f$.

Theorem 4 Let $f:(X, \partial X) \rightarrow(X, \partial X)$ be a map in reduced form. Then $f$ may be homotoped to a map in reduced form with the property that every simple closed curve in the inverse image of $A$ is of type $(0,1)$.

Proof We claim that all the types of simple closed curves listed in Lemma 2 except $(0,1)$ may be excluded as a component of the inverse image of $A$ for some map in reduced form homotopic to the given map. Suppose $K$ is a component of $f^{-1}(A)$ of type $(1,0)$ or type $(1,1)$. Then there is a closed neighborhood $N$ of $K$ such that $N \cap f^{-1}(A)=K$ and $N-K$ is connected. Since $f(N-K)$ is a connected subset of $X-A$, it lies in one of the three components of $X-A$, each of which is contractible. We denote the component of $X-A$ that contains $f(N-K)$ by $B$. Since $f$ maps the boundary $\partial N$ of $N$ into the contractible set $B$, we can extend $f \mid \partial N$ to a map $g: N \rightarrow B$. Extend $g$ to all of $X$ by letting it equal $f$ outside of $N$. Then $g$ is a reduced form of $f$ with $g^{-1}(A)=f^{-1}(A)-K$. Repeating this procedure a finite number of times, we may assume we have a reduced form for $f$ (which we still call $f$ ) in which no component of $f^{-1}(A)$ is a simple closed curve of type $(1,0)$ or type $(1,1)$. Since $f$ is in reduced form, if $\lambda$ is an arc in $A$ with endpoints in $f^{-1}(A)$ and interior disjoint from $f^{-1}(A)$, then the endpoints of $\lambda$ must be mapped to distinct components of $A$. We claim that this property implies that simple 
closed curves of types $(2,0),(2,1)$ and $(2,2)$ cannot be components of $f^{-1}(A)$. To prove it, let $K \subseteq f^{-1}(A)$ be an innermost simple closed curve of type $(2, n)$ for any $n=0,1,2$, then $f(K)$ is connected so it lies entirely inside one of the $A_{j}$. Taking the two points of intersection of $K$ with $A_{1}$, the arc $\lambda \subseteq A_{1}$ between them would have no points of the inverse image of $A$ in its interior because there are now no simple closed curves of types $(1,0)$ or $(1,1)$. But the endpoints of $\lambda$ map to the same component of $A$, contrary to the definition of reduced form. Since we have eliminated from the inverse image of $A$ all simple closed curves of types $(0,1),(1,1),(2,0),(2,1)$ and $(2,2)$, we conclude that, if there are any simple closed curves, they must be of type $(0,1)$.

In order to complete the analysis of the components of $f^{-1}(A)$ for $f$ in reduced form, we turn now to the isotopy classification of properly embedded arcs in $X$. Recall that the components of $\partial D-\left(H_{1} \cup H_{2}\right)$ are $T_{1} \cup T_{2} \cup T_{3} \cup T_{4}$ where $T_{2} \subset C_{2}$, as in Figure 1. If a properly embedded arc $K$ is isotopic to a line segment in $D$ with endpoints in $T_{i}$ and $T_{j}$, then we will call $K$ an arc of type $[i, j]$.

We begin with the classification of $\operatorname{arcs} K$ whose endpoints lie in different components of $\partial X$.

Theorem 5 Let $K$ be an arc in $X$ that has minimal intersection with $A$ in its isotopy class and whose endpoints lie in different components of $\partial X$. Then $K$ is of type $[1,2]$ or of type $[2,3]$ and $K$ lies entirely in $D$.

Proof We can isotope $K$ so that one endpoint lies in $T_{1} \cup T_{3} \cup T_{4}$ and the other in $T_{2}$ and $K$ has minimal intersection with $A$ within its isotopy class. It will be convenient to orient $K$ so that we view $K$ as ending in $T_{2}$. We will first show that the first intersection of $K$ with $H_{1} \cup H_{2}$ cannot be with $H_{1}$. The intersections of $K$ with $\partial D$ cannot begin $T_{1} A_{1} A_{2}$ because $K$ could be isotoped to begin at $T_{4}$, so the intersection with $A$ is not minimal. In the same way, the intersection $T_{1} A_{2} A_{1}$ can be isotoped to begin at $T_{3}$. If the intersections began with $T_{3} A_{1} A_{2}$ we can isotope to begin at $T_{1}$ whereas, if it began with $T_{3} A_{2} A_{1}$, the arc could not reach $T_{2}$. Checking the two corresponding cases for $K$ beginning in $T_{4}$, we conclude that if $K$ intersects $H_{1} \cup H_{2}$, it must first intersect $H_{2}$. If $K$ starts in $T_{1}$, intersects $H_{2}$ and then $H_{1}$, we can assume without loss of generality that the intersections are $T_{1} A_{3} A_{4} A_{1} A_{2}$, but then $K$ cannot end in $T_{2}$. On the other hand if, after intersecting $H_{2}$, the arc $K$ does not next intersect $H_{1}$, then it must have its last intersection with $H_{2}$. But it is clear that, if the last intersection is with $H_{2}$, we can isotope $K$ to reduce the 
intersection with $A$, so that intersection was not minimal. Thus, if $K$ starts at $T_{1}$ and has minimal intersection with $A$, then it does not intersect $A$ at all. If $K$ starts at $T_{3}$ and intersects $A$ in the order $T_{3} A_{3} A_{4}$, it also would have its last intersection with $\mathrm{H}_{2}$ and thus not have minimal intersection with $A$. The intersection with $A$ is also not minimal if $K$ begins $T_{3} A_{4} A_{3}$ because the first intersection can be reduced by isotoping $K$ to begin in $T_{4}$. Symmetric arguments when $K$ begins in $T_{4}$ lead us to conclude that, no matter where $K$ begins, it cannot intersect $H_{1} \cup H_{2}$ and so $K$ lies entirely in $D$. Therefore $K$ is isotopic to a line segment ending in $T_{2}$ and beginning in either $T_{1}, T_{3}$ or $T_{4}$. Since the line segment between $T_{2}$ and $T_{4}$ is isotopic to the line segment between $T_{2}$ and $T_{3}$, there are just the two possibilities given.

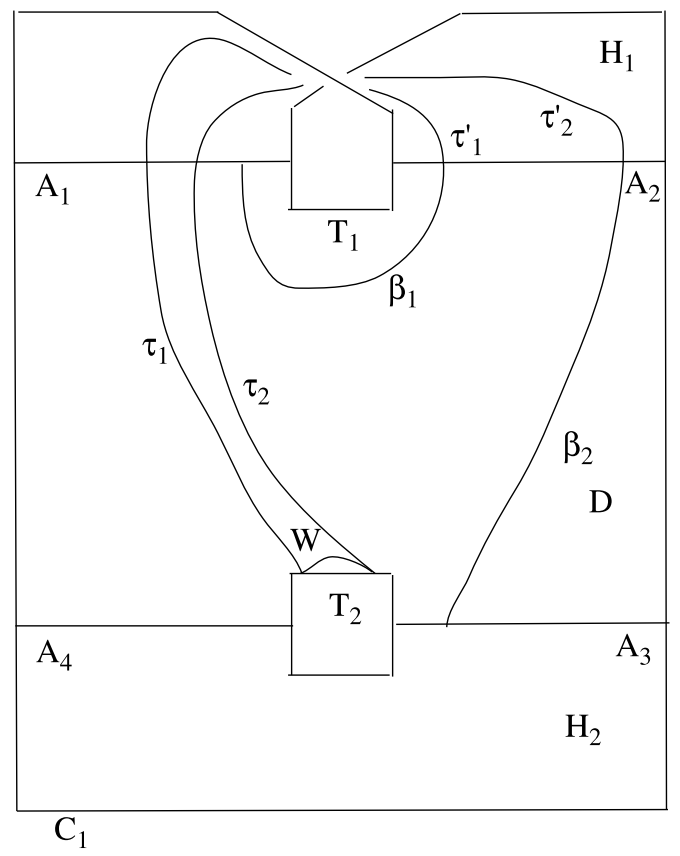

Figure 3

Lemma 4 Let $K$ be an arc in $X$, both of whose endpoints lie in $C_{2}$, such that the number of points in $K \cap A$ is minimal in the isotopy class of $K$. Let $W$ be an arc in $D$ connecting the endpoints of $K$ and otherwise disjoint from $K$. Then the number of points in $(K \cup W) \cap A$ is minimal in the isotopy class of the simple closed curve $K \cup W$.

Proof Let $\tau_{1}$ and $\tau_{2}$ be the two components of $K \cap D$ that intersect $C_{2}$ (see 
Figure 3). Assuming, without loss of generality, that $\tau_{1}$ intersects $A_{1}$. We note that the minimality condition on $K \cap A$ also assures us that $\tau_{2}$ cannot intersect $A_{3}$ or $A_{4}$. Suppose $\tau_{2}$ also intersects $A_{1}$. Consider those portions of $K$, which we denote by $\tau_{i}^{\prime}$ for $i=1,2$, that start at the endpoints in $A_{1}$ of the respective $\tau_{i}$ and that intersect the $A_{i}$ in the same order, and that are maximal with respect to this property. Figure 3 illustrates the case in which in the $\tau_{i}^{\prime}$ intersect in the order $A_{1} A_{2}$. The other possible case, in which the order is $A_{1} A_{2} A_{3} A_{4} A_{1} A_{2}$, can be described by a similar figure. Let $\beta_{i}$ denote the components of $K-A$ whose closures intersect the $\tau_{i}^{\prime}$. Since, by definition of the $\tau_{i}^{\prime}$, the arcs $\beta_{1}$ and $\beta_{2}$ cannot have their endpoints in the same $A_{i}$, one must have an endpoint in $A_{1}$ and the other an endpoint in $A_{3}$, as we illustrated in Figure 3. Relative to those endpoints, we can isotope the portion of $K \cup W$ consisting of $W \cup \tau_{1} \cup \tau_{2} \cup \tau_{1}^{\prime} \cup \tau_{2}^{\prime} \cup \beta_{1} \cup \beta_{2}$ to an arc in $D$ with endpoints in $A_{1}$ and $A_{3}$. Thus, if we isotope $K \cup W$ so that its intersection with $A$ is minimal, we obtain a simple closed curve that contains such an arc. However, as we can see in Figure 2, none of the simple closed curves that have minimal intersection with $A$ can contain an arc in $D$ connecting $A_{1}$ and $A_{3}$. Thus, assuming that $\tau_{2}$ intersects $A_{1}$ has lead to a contradiction and we conclude that $\tau_{2}$ intersects $A_{2}$, which implies that the number of points in $(K \cup W) \cap A$ is minimal in the isotopy class of the simple closed curve $K \cup W$.

The punctured Möbius band $X$ can be constructed from a surface $\tilde{X}$ that consists of the two-dimensional sphere with four symmetrically-placed open discs deleted, by identifying antipodal points. Consider a 90-degree rotation of $\widetilde{X}$ that is equivariant with respect to the antipodal action. Then, for $\phi: X \rightarrow X$ the corresponding homeomorphism, we have $\phi\left(C_{2}\right)=C_{1}$ and $\phi\left(C_{1}\right)=C_{2}$. Moreover, we may assume that $\phi\left(x_{1}\right)=x_{2}$ then, since $\phi^{2}$ is the identity map, we have $\phi\left(x_{2}\right)=x_{1}$ as well.

Lemma 5 Let $K$ be an arc in $X$ both of whose endpoints lie in the same component of $\partial X$. If both endpoints are in $C_{2}$, then $K$ is isotopic to an arc obtained by removing an arc from a simple closed curve of type $(1,0),(2,0)$ or $(2,1)$ and connecting the endpoints of the arc that remains to $C_{2}$ by parallel arcs. Such arcs are said to be of types $\{1,0\},\{2,0\}$ and $\{2,1\}$, respectively. If both endpoints are in $C_{1}$, then either $K$ is of type [1,3] or of type [3,4], or it is isotopic to an arc with both endpoints in $T_{1}$ that passes once through $\mathrm{H}_{2}$ and does not intersect $H_{1}$. Such an arc is said to be of type $\{0,1\}$.

Proof Suppose both endpoints of $K$ are in $C_{2}$. Isotope $K$ so that the number of points in $K \cap A$ is minimal in the isotopy class of $K$. Let $W$ be an arc in 
$D$ connecting the endpoints of $K$ and otherwise disjoint from $K$. By Lemma 4, the number of points in $(K \cup W) \cap A$ is minimal in the isotopy class of the simple closed curve $K \cup W$ and therefore $K \cup W$ is of one of the six types of simple closed curves described by Lemma 2. Thus, once the arc $K$ has been isotoped so that the number of points in $K \cap A$ is minimal in the isotopy class, it must have the same intersections with $H_{1}$ and $H_{2}$ as in that lemma. We can describe $K$ itself by deleting an arc in $D$ from a simple closed curve of Lemma 2 and connecting the endpoints of what remains with $C_{2}$ by parallel arcs. It is easy to check that, if we carry out this construction with the types $(0,1),(1,1)$ or $(2,2)$, then $K \cap A$ is not minimal in the isotopy class of $K$. That leaves the three types $(1,0),(2,0)$ and $(2,1)$; see Figure 4.

Now suppose both endpoints of $K$ are in $C_{1}$. Then the endpoints of $\phi(K)$ are in $C_{2}$ and so $\phi(K)$ is isotopic to some arc $K^{\prime}$ that is one of the three types just described. Applying $\phi=\phi^{-1}$ once more, we have $K$ isotopic to $\phi\left(K^{\prime}\right)$ and thus there are three isotopy types of arcs with both endpoints in $C_{1}$. To show that those three isotopy types are the ones described in the statement of the theorem, it suffices to prove that no two such arcs are boundary-preserving isotopic. Proper arcs are relative 1-cycles that represent classes of $H_{1}(X, \partial X)$. If two properly embedded arcs are boundary-preserving isotopic, then the corresponding cycles are homologous. However, the arcs of type $[1,3],[3,4]$ and $\{0,1\}$ represent three different elements of $H_{1}(X, \partial X)$.

In the proof of Lemma 5, we used the homeomorphism $\phi$, that interchanges the components of $\partial X$, in order to apply our analysis of the isotopy classes of arcs with endpoints in $C_{2}$ to arcs whose endpoints lie in $C_{1}$. We will also use $\phi$ so that information about minimizing the number of fixed points through a homotopy can be extended to additional cases by means of the following, easily verified, result.

Lemma 6 Let $f:(X, \partial X) \rightarrow(X, \partial X)$ be a map and suppose that $g:(X, \partial X)$ $\rightarrow(X, \partial X)$ is homotopic, as a map of pairs, to $\phi f \phi$, then $\phi g \phi$ is homotopic to $f$ and the set of fixed points of $\phi g \phi$ is homeomophic to the set of fixed points of $g$.

If a map $f:(X, \partial X) \rightarrow(X, \partial X)$ is in reduced form, then for $i=1,2$, the map $f_{i}: C_{i} \rightarrow C_{i \#}$ is either the constant map at $x_{i \#} \notin A$ if $\left|d_{i}\right|=0$ or, otherwise, $f_{i}$ is a $\left|d_{i}\right|$-to-one map. If $\left|d_{i}\right|=0$, then $f_{i}^{-1}(A) \cap C_{i}$ is the empty set.

Definition Suppose that $\left|d_{i}\right| \neq 0$ then, for each point of the finite set $f_{i}^{-1}(A) \cap$ $C_{i}$ we record the integer $1,2,3$ or 4 corresponding to the component of $A$ to 
which it is sent by $f$. As we go once around the simple closed curve $C_{i}$, we obtain an ordered set made up of the integers 1 through 4 which we call the pattern of $f_{i}$. Of course the pattern depends on where on $C_{i}$ we begin our circuit of the simple closed curve and in which direction we go.

Lemma 7 Given a map $f:(X, \partial X) \rightarrow(X, \partial X)$ in reduced form, if the degree $\left|d_{i}\right|$ of $f_{i}: C_{i} \rightarrow C_{i \#}$ is non-zero, then we may choose the starting point and direction so that the pattern of $f_{i}$ is (121234) $)^{\left|d_{i}\right|}$ if $i^{\#}=1$ and $(34)^{\left|d_{i}\right|}$ if $i^{\#}=2$.

Proof Since $C_{1}$ intersects $A$ in six points then, if $i^{\#}=1$, it must be that $f_{i}^{-1}(A) \cap C_{i}$ consists of $6\left|d_{i}\right|$ points because $f$ is in reduced form. Moreover, by starting at a suitable place in $C_{i}$ and choosing the correct direction, the points of $f_{i}^{-1}(A) \cap C_{i}$ that we encounter will be first mapped to $A_{1}$, then to $A_{2}$, next to $A_{1}$ again and so on to produce the pattern $(121234)^{\left|d_{i}\right|}$ (see Figure 1). On the other hand, $C_{2}$ intersects $A$ only at one point each of $A_{3}$ and $A_{4}$. Therefore, if $i^{\#}=2$ then, choosing the correct starting point and direction, the pattern in this case will be $(34)^{\left|d_{i}\right|}$.

Theorem 6 Let $f:(X, \partial X) \rightarrow(X, \partial X)$ be a map in reduced form such that $f_{1}$ is essential, then every proper arc in $f^{-1}(A)$ is one of the following types: $[1,2],[1,3],[2,3]$ or $\{1,0\}$.

Proof We first show that there are no arcs of type $[3,4]$ in $f^{-1}(A)$. Suppose that there are and let $K$ by the "innermost" such arc. That is, letting $L$ be the component of $X-K$ that contains $C_{2}$, then no other arc of that type is in $L$. Arcs that are components of $f^{-1}(A)$ that lie in $L$ must be of type $[2,3]$. Let $f(K)=A_{j}$. If there were no arcs of type $[2,3]$ in $L$, the pattern of $f_{1}$ would contain $j j$, contrary to the Lemma 7 . Thus there must be type $[2,3]$ arcs in $L$ and these are the only components of $f^{-1}(A)$ that intersect $C_{2}$. Therefore the number of type $[2,3]$ arcs is either $6\left|d_{2}\right|$ or $2\left|d_{2}\right|$, depending on whether $C_{2}$ is mapped to $C_{1}$ or to $C_{2}$. But that is an even number in either case whereas, by Lemma 7 , the pattern of $f_{1}$ is either $(121234)^{d_{1}}$ or $(34)^{\left|d_{1}\right|}$. A contradiction occurs because, in either possible pattern for $f_{1}$, the number of integers between any repetition of an integer $j$ is odd. Thus there are no arcs of type $[3,4]$. Basically the same argument demonstrates that arcs of $\{0,1\}$ cannot be components of $f^{-1}(A)$, with arcs of type $[1,2]$ taking the role of the arcs of type $[2,3]$ in the previous argument.

We next eliminate arcs of $\{2,0\}$ as possible components of $f^{-1}(A)$. Suppose there are arcs of this type and let $K$ denote the "innermost" such component. 
That is, there is an $\operatorname{arc} \alpha$ in $C_{2}$ connecting the endpoints of $K$, and with its interior in the component of $X-K$ that does not contain $C_{1}$, such that no other arc of type $\{0,2\}$ intersects $\alpha$. Let $f(K)=A_{j}$. If there were no other component of $f^{-1}(A)$ with endpoints in the interior of $\alpha$, then the pattern of $f_{2}$ would contain $j j$, contrary to Lemma 7 . However, a component of $f^{-1}(A)$ with endpoints in $\alpha$ would be of type $\{1,0\}$ and the innermost of these would, in the same way, produce a pattern for $f_{2}$ contrary to Lemma 7 . Thus there are no arcs of type $\{2,0\}$ that are components of $f^{-1}(A)$.

The proof that there are no components of $f^{-1}(A)$ of type $\{2,1\}$ is similar to the argument for type $\{2,0\}$. This time let $K$ be the "outermost" component of type $\{2,1\}$. This means that there is an $\operatorname{arc} \beta$ in $C_{2}$ connecting the endpoints of $K$, and with its interior in the component of $X-K$ that does not contain $C_{1}$, such that no other arc of type $\{2,1\}$ has its endpoints in $\beta$. If there were no components of $f^{-1}(A)$ intersecting the interior of $\beta$, the pattern of $f_{2}$ would again contain $j j$ where $f(K)=A_{j}$, contrary to Lemma 7 . If there were such components, however, they would be of type $\{1,0\}$ and the "outermost" of these would lead to the same contradiction. Thus there are no components of $f^{-1}(A)$ that are arcs of type $\{2,1\}$. By Theorem 5 and Lemma 5 , the four types listed in the statement of the theorem are the only possibilities remaining (see Figure 4).

\section{Boundary inessential maps}

Definition Represent the points of the unit circle $S^{1}$ by $e^{i \theta}$ for $0 \leq \theta \leq 2 \pi$. Let $R$ be an annulus embedded in $X$ and $\alpha$ an arc in $R$ such that there is a homeomorphism $\psi:[0,1] \times S^{1} \rightarrow R$ with $\psi\left([0,1] \times\left\{e^{i 0}=1\right\}\right)=\alpha$. Given a map $g: \alpha \rightarrow X$, the annulus extension of $g$ is the map $g: R \rightarrow X$ defined by $g\left(\psi\left(t, e^{i \theta}\right)\right)=g(\psi(t, 1))$ for all $0 \leq t \leq 1$ and $0 \leq \theta \leq 2 \pi$.

Theorem 7 If $f:(X, \partial X) \rightarrow(X, \partial X)$ is boundary inessential, then $M F_{\partial}[f]=$ $N_{\partial}(f)$.

Proof By Lemma 3, we may assume that $f$ is in reduced form. Therefore the restriction of $f$ to each component $C_{j}$ is a constant map to one of the points $x_{1}$ or $x_{2}$; we write $f\left(C_{j}\right)=x_{j \#}$. Consequently, the components of $f^{-1}(A)$ must be simple closed curves that, by Theorem 4, we may assume are of type $(0,1)$. See Figure 5 . We denote the components by $K_{1}, \ldots, K_{m}$ where 

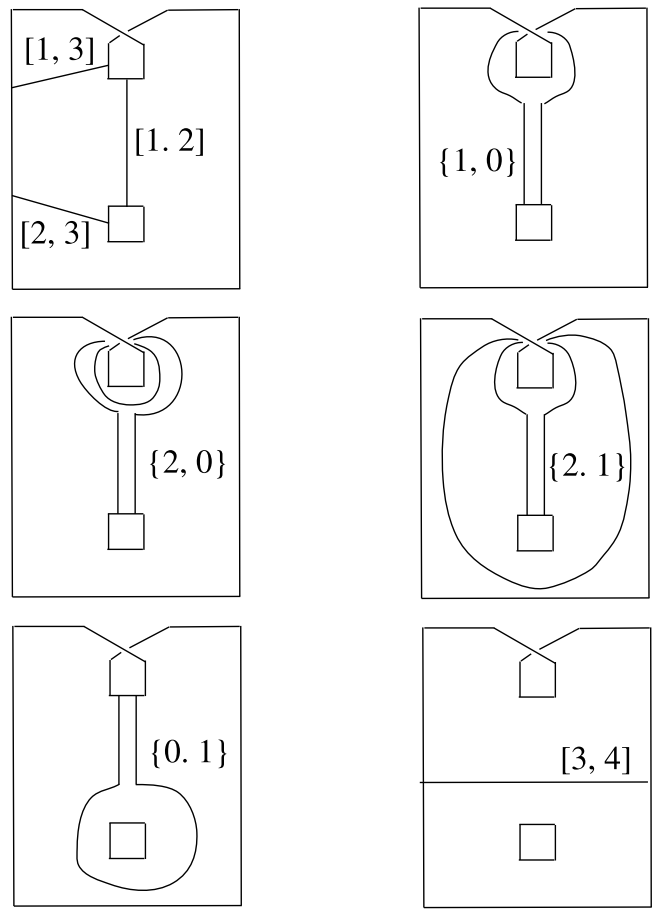

Figure 4

$K_{i+1}$ is the component of $X-K_{i}$ containing $C_{2}$ and $K_{i}$ and $K_{i+1}$ bound an annulus $R_{i}$ that contains no other component of $f^{-1}(A)$. We will describe a map $g:(X, \partial X) \rightarrow(X, \partial X)$ which is boundary-preserving homotopic to $f$, is also constant on each component of $\partial X$, and that has a single fixed point in the interior of $X$ if $\operatorname{Im}_{\partial}(f)=2$ and $f\left(C_{1}\right)=C_{2}$. In that case, $g$ has no fixed points on $\partial X$ and, since its Lefschetz number is nonzero, we have $M F_{\partial}[f]=N_{\partial}(f)$. Otherwise, $g$ will have no fixed points in the interior of $X$ so obviously $M F_{\partial}[f]=N_{\partial}(f)$.

Let $\left[x_{1}, x_{2}\right]$ denote the line segment and let $h_{1}$ and $h_{2}$ be the simple closed curves shown in Figure 5.

We define a map $g^{\prime}$ that sends all of $X$ to $G=\left[x_{1}, x_{2}\right] \cup h_{1} \cup h_{2}$. Let $R$ be the annulus in $X$ bounded by the simple closed curves $h_{2}$ and $C_{2}$. We map the closure of $X-R$ to the line segment $\left[x_{0}, x_{1 \#}\right]$ by sending $h_{2}$ to $x_{0}$ and extending. We will obtain $g^{\prime}$ on $R$ by defining a map on the line segment $\left[x_{0}, x_{2}\right]$ and then extending to all of $R$ by using annulus extensions. The behavior of the intersection of $\left[x_{0}, x_{2}\right]$ with each $R_{i}$ will determine how $g^{\prime}$ is defined on $R_{i}$. We must send $x_{2}$ to $x_{2 \#}=f\left(C_{2}\right)$, we let $g^{\prime}\left(x_{0}^{\prime}\right)=x_{0}$ and we extend linearly 
to the line segment $\left[x_{2}, x_{0}^{\prime}\right]$. Now each annulus $R_{i}$ is mapped by $f$ either to $D$ or to one of the handles $H_{j}$ and, for adjacent annuli $R_{i}$ and $R_{i+1}$, one must go to $D$ and the other to a handle. For each $R_{i}$ mapped to $D$, choose a point $x_{i}$ in the interior of the line segment $R_{i} \cap\left[x_{0}, x_{0}^{\prime}\right]$. For each such $i$, the map $g^{\prime}$ sends the line segment $\left[x_{i}, x_{i+2}\right]$ once around $h_{1}$ or once around $h_{2}$, with $x_{i}$ and $x_{i+2}$ sent to $x_{0}$, depending on whether $f$ maps $R_{i+1}$ to $H_{1}$ or to $H_{2}$. Annulus extension on each $R_{i}$ completes the definition of $g^{\prime}$.

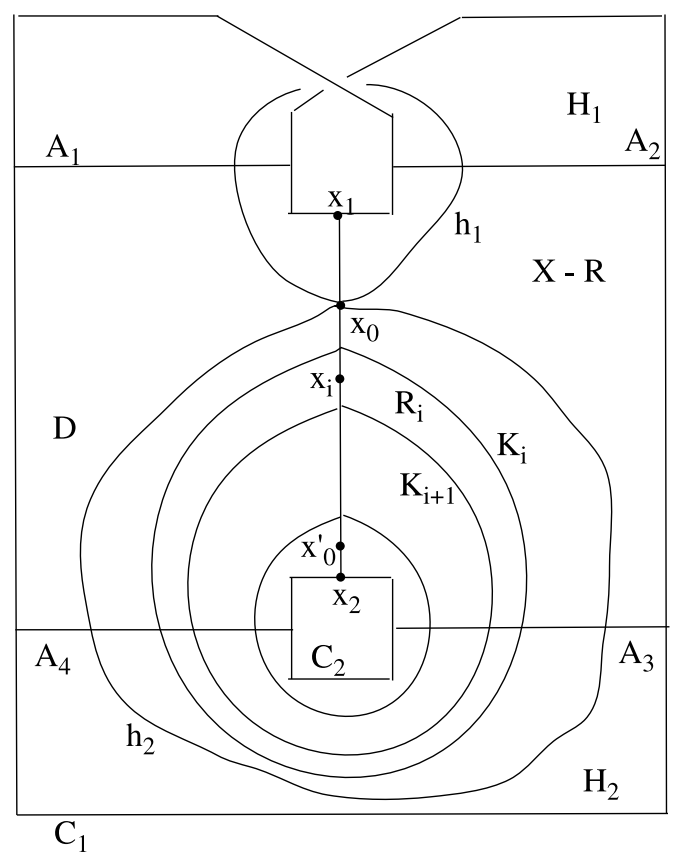

Figure 5

If $f\left(C_{1}\right)=x_{1}$, then $g^{\prime}$ is the identity on the line segment $\left[x_{0}, x_{1}\right]$ but there are no other fixed points on the interior of $X$. Let $G^{\prime}=\left[x_{1}, x_{2}\right] \cup h_{1}^{\prime} \cup h_{2}^{\prime}$ where $h_{i}^{\prime}$ is isotopic to $h_{i}$ and intersects $\left[x_{1}, x_{2}\right]$ only at $x_{1}$. Let $\rho: G \rightarrow G^{\prime}$ map $\left[x_{1}, x_{2}\right]$ to itself, taking $\left[x_{1}, x_{0}\right]$ to $x_{1}$ and extending linearly over $\left[x_{0}, x_{2}\right]$, and sending each $h_{i}$ to $h_{i}^{\prime}$ by a homeomorphism. Note that $\rho g^{\prime}$ is homotopic to $f$ as a map of the pair $(X, \partial X)$. If $f\left(C_{1}\right)=x_{1}$ and $f\left(C_{2}\right)=x_{2}$, then $x_{1}$ and $x_{2}$ are the only fixed points of $g=\rho g^{\prime}$. If $f\left(C_{1}\right)=f\left(C_{2}\right)=x_{1}$, then $g=\rho g^{\prime}$ has a fixed point only at $x_{1}$. Now suppose $f\left(C_{1}\right)=f\left(C_{2}\right)=x_{2}$ then, by Lemma 6 , the map $g=\phi \rho g^{\prime} \phi$ is homotopic to $f$ and its only fixed point is $x_{2}$. Finally, if $f\left(C_{1}\right)=x_{2}$ and $f\left(C_{2}\right)=x_{1}$, then we can set $g=g^{\prime}$ because $g^{\prime}$ will have the required property, namely, one fixed point in the interior of $X$, at $x_{0}$. 


\section{$3 \quad$ Boundary essential maps}

Theorem 8 If $f:(X, \partial X) \rightarrow(X, \partial X)$ is a boundary essential map such that $\operatorname{Im}_{\partial}(f)=2$, then $N_{\partial}(f)=M F_{\partial}[f]$.

Proof Suppose for now that $f\left(C_{i}\right)=C_{i}$ for $i=1,2$. Throughout the proof, homotopies will be understood to be relative to $\partial X$. We will show that $f$ maps $X$ onto itself. Let $Z=\partial X \cup A_{1} \cup A_{3}$. Suppose $f$ is not onto, then we may assume there is a point of $X-Z$ that is not in the image of $f$. By retracting, there is a map homotopic to $f$ whose image is $Z$. We use [3], to obtain a map $g: X \rightarrow Z$ such that, for $i=1,2$, the set $L_{i}=g^{-1}\left(x_{i}\right)$ is a 1-dimensional subpolyhedron of $X$. There is a component $\alpha_{i}$ of $L_{i}$ that is an essential properly embedded arc or an essential simple closed curve intersecting $C_{i}$ in a single point or else connected to $C_{i}$ by an arc meeting $C_{i}$ in a point. If no such $\alpha_{i}$ existed, there would be a simple closed curve in the complement of $L_{i}$ that is isotopic to $C_{i}$. But that would imply that $f_{i}$ : $C_{i} \rightarrow C_{i}$ is inessential, contrary to the hypotheses. If $\alpha_{1}$ and $\alpha_{2}$ are both essential properly embedded arcs, then it follows from Lemma 5 that $\alpha_{1} \cap \alpha_{2} \neq \emptyset$ (see Figure 4). If one or both of the $\alpha_{i}$ is a simple closed curve intersecting $\partial X$ in one point or connected to it by an arc and it were true that $\alpha_{1} \cap \alpha_{2}=\emptyset$, then there would be nearby properly embedded arcs with the same property, contrary to Lemma 5, so we conclude that $\alpha_{1} \cap \alpha_{2} \neq \emptyset$. But $L_{1} \cap L_{2}=\emptyset$ by definition so the contradiction proves that $f$ maps onto $X$. Every map of $X$ that is homotopic to $f$ through boundary-preserving homotopies is boundary-essential and therefore onto, as we have shown, so the geometric degree of $f$ is nonzero. Consequently, by a theorem of Edmonds (see 14, Theorem 1.1 for a corrected version of the result), $f$ is homotopic to $q p$ where $p: X \rightarrow Y$ is a pinch and $q: Y \rightarrow X$ is an $n$-fold branched cover. Triangulate $X$ so that the branch points are vertices and let $v$ be the number of vertices in the triangulation. Triangulate $Y$ so that $q$ is a simplicial map, then the number of vertices of this triangulation of $Y$ is $n v-b$ for some $b \geq 0$ depending on the branching. Therefore, the Euler characteristic of $Y$ is $\chi(Y)=n \chi(X)-b=-n-b<0$. If $p$ were a nontrivial pinch, then there would be a subsurface $P$ of $X$, not a disc, such that $\partial P$ is connected and $Y=X / P$. But that would imply $\chi(Y)=\chi(X)-\chi(P)+1=$ $-\chi(P) \geq 0$ so the pinch must be trivial, that is, $Y=X$ and $p$ is the identity map. But then we have $-1=-n-b$ which implies that $b=0$ and $n=1$, that is, $q$ is a homeomorphism. It follows by [7] that $N_{\partial}(q)=M F_{\partial}[q]$ and therefore, since $f$ is homotopic to $q$, that $N_{\partial}(f)=M F_{\partial}[f]$ when $f\left(C_{i}\right)=C_{i}$ for $i=1,2$. Now suppose $f\left(C_{1}\right)=C_{2}$ and $f\left(C_{2}\right)=C_{1}$. Let $\phi: X \rightarrow X$ be the homeomorphism that interchanges $C_{1}$ and $C_{2}$. The map $\phi f$ has the property 
that $\phi f\left(C_{i}\right)=C_{i}$ for $i=1,2$ so, as we have just demonstrated, $\phi f$ is homotopic to a homeomorphism $q$. But then $f$ is homotopic to the homeomorphism $\phi q$ so we may again apply [7] to complete the proof.

Theorem 9 If $f:(X, \partial X) \rightarrow(X, \partial X)$ is a boundary essential map such that $\operatorname{Im}_{\partial}(f)=1$, then $N_{\partial}(f)=M F_{\partial}[f]$.

Proof We first consider the case that $f(\partial X)=C_{2}$. We claim that $f$, assumed to be in reduced form, is homotopic modulo the boundary to a map $g: X \rightarrow C_{2}$ which agrees with $f$ on $\partial X$ and that would establish $N_{\partial}(f)=M F_{\partial}[f]$ in this case. Since $f$ is an essential map on $C_{2}$, there are components of $f^{-1}(A)$ that are arcs with at least one endpoint in $C_{2}$. No simple closed curve of type $(0,1)$ can lie in the complement of such an arc; see Figure 4. Thus there are no simple closed curves in $f^{-1}(A)$ since, by Theorem 4 , we need consider only those of type $(0,1)$. Each arc $K$ that is a component of $f^{-1}(A)$ is mapped by $f$ in such a way that the endpoints of $K$ are mapped to the same point of $C_{2} \cap A$; call that point $a_{j}$. We let $g$ agree with $f$ on $\partial X$ and define $g$ on each component $K$ of $f^{-1}(A)$ by defining $g(K)=a_{j}$ where $f(K) \subseteq A_{j}$ for $j=3,4$. Each component $V$ of $X-\left(f^{-1}(A) \cup \partial X\right)$ is a disk bounded by components of $f^{-1}(A)$ and arcs in $\partial X$. Thus $g$ has been defined to send the simple closed curve $\partial V$ to $C_{2}$ and it is homotopic to $f$ on $\partial V$ where $f$ extends to $V$. Since the inclusion of $C_{2}$ into $X$ induces a monomorphism of the fundamental groups, the degree of $g$ on $\partial V$ is zero and therefore it extends to a map of $V$ to $C_{2}$. In this way we define $g: X \rightarrow C_{2}$ as we claimed. If $f(\partial C)=C_{1}$, we can apply the first part of the proof to $\phi f \phi$ to obtain $g: X \rightarrow C_{2}$. By Lemma 6 , the map $\phi g \phi: X \rightarrow C_{1}$ establishes that $N_{\partial}(f)=M F_{\partial}[f]$ in this case.

\section{Boundary semi-essential maps}

Lemma 8 If $f:(X, \partial X) \rightarrow(X, \partial X)$ is a boundary semi-essential map where $f\left(C_{1}\right)=C_{1}$ and $f_{1}$ is essential, then $N_{\partial}(f)=M F_{\partial}[f]$.

Proof We assume $f$ is in reduced form then, since $f_{2}$ is inessential, either $f\left(C_{2}\right)=x_{1} \in T_{1}$ or $f\left(C_{2}\right)=x_{2} \in T_{2}$ so $f^{-1}(A)$ does not intersect $C_{2}$. By Theorem 6 , there are just four possible types of arcs that can be components of $f^{-1}(A)$ and only those of type $[1,3]$ fail to intersect $C_{2}$. By Theorem 4, the only type of simple closed curve that could be a component of $f^{-1}(A)$ is $(0,1)$. Referring to Figure 6 , the complement of the line segment $\left[y_{1}, y_{2}\right]$ has 
two components that we call $U$ and $L$, where $C_{2} \subset L$. All the simple closed curves in $f^{-1}(A)$ are contained in the component of the complement of $h_{2}$ that contains $C_{2}$. We have chosen a point $z$ in $U \cap C_{1}$ such that the line segment $\left[x_{0}, z\right]$ does not intersect $f^{-1}(A)$. Since all the points of $f^{-1}(A) \cap C_{1}$ lie in $U$, we may modify $f$ so that it maps all of $L \cap C_{1}$ to $z$, without adding any fixed points to $f_{1}$. The points $x_{3}$ and $x_{4}$ on the line segment between $x_{0}$ and $x_{2}$ are chosen so that the segments $\left[x_{0}, x_{3}\right]$ and $\left[x_{2}, x_{4}\right]$ are disjoint from $f^{-1}(A)$. We will construct a map $g:(X, \partial X) \rightarrow(X, \partial X)$ that is homotopic to $f$ as a map of pairs and identical to $f$ on the boundary of $X$ such that $g$ has no fixed points on the interior of $X$. We obtain $g$ on $L$ by defining it on the line segment $\left[x_{0}, x_{2}\right]$ and then, using annulus extensions, we extend it to all of $L$. We introduce notation as follows. Letting $h=h_{1} \cup h_{2}$ we set $a_{i}=h \cap A_{i}$. We number the simple closed curves of $f^{-1}(A)$ as $K_{1}, \ldots, K_{m}$ where $K_{j+1}$ is in the component of $X-K_{j}$ that contains $C_{2}$ and we set $k_{j}=K_{j} \cap\left[x_{0}, x_{2}\right]$. We define $g:\left[x_{0}, x_{2}\right] \rightarrow\left[z, x_{0}\right] \cup\left[x_{0}, x_{2}\right] \cup h$ in the following way. Let $g\left(x_{0}\right)=z$ and extend to $\left[x_{0}, x_{3}\right]$ as a homeomorphism onto $\left[z, x_{0}\right]$. For $j=1, \ldots, m$ we have $f\left(K_{j}\right) \subseteq A_{i(j)}$ for some $1 \leq i(j) \leq 4$ and we define $g\left(k_{j}\right)=a_{i(j)}$. We then define $g$ on $\left[x_{3}, k_{1}\right]$ by sending it to the arc in $h$ connecting $x_{0}$ to $a_{i(1)}$ and on each $\left[k_{j}, k_{j+1}\right]$ by mapping to an arc in $h$ that connects $a_{i(j)}$ to $a_{i(j+1)}$ through the component of $X-A$ that contains $f\left(k_{j}, k_{j+1}\right)$. Setting $g\left(x_{4}\right)=x_{0}$, we define $g$ on $\left[k_{m}, x_{4}\right]$ by mapping onto the arc in $h$ that connects $a_{i(m)}$ to $x_{0}$. Finally, $g$ maps $\left[x_{4}, x_{2}\right]$ homeomorphically onto $\left[x_{0}, f\left(C_{2}\right)\right]$. We note that $g$ will have a fixed point at $x_{2}$ in the case that $f\left(C_{2}\right)=x_{2}$ and no fixed points if $f\left(C_{2}\right)=x_{1}$. Choose two simple closed curves in $L$ in the complement of the $K_{i}$ intersecting $\left[x_{0}, x_{2}\right]$ in $x_{3}$ and $x_{4}$ respectively. If we delete these simple closed curves as well as all the $K_{i}$ from $L$, then the closure of each component is an annulus containing a segment of $\left[x_{0}, x_{2}\right]$. Applying annulus extensions to each of these, we now have $g$ defined on $L$.

The map $g$ is defined on $U$ as follows. Each component of $f^{-1}\left(A_{3} \cup A_{4}\right)$ has both its endpoints mapped a single point, either $C_{1} \cap A_{3}$ or $C_{1} \cap A_{4}$. Consider a component $K$ of $f^{-1}\left(A_{1} \cup A_{2}\right)$ that is adjacent to a component $K^{\prime}$ of $f^{-1}\left(A_{3} \cup A_{4}\right)$. As the image of the two arcs of $C_{1}$ between $K$ and $K^{\prime}$ must map to the same arc of $D \cap C_{1}$, then the endpoints of $K$ must map to the same point, either $T_{3} \cap A_{1}$ or $T_{4} \cap A_{2}$. Next, for a component of $f^{-1}(A)$ adjacent to either $K$ or $K^{\prime}$, its endpoints must also map to a single point by the same argument. Continuing in this manner, we conclude that both endpoints of any component $\mathrm{K}$ of $f^{-1}(A)$ are sent by $f$ to the same point $a_{K} \in A \cap C_{1}$. Letting $g(K)=a_{K}$ for each component $K$ of $f^{-1}(A)$ then, noting that $g\left(\left[y_{1}, y_{2}\right]\right)=z$, we can extend to all of $U$ so that $g(U) \subseteq C_{1}$ and $g^{-1}(A)=f^{-1}(A)$. Since 


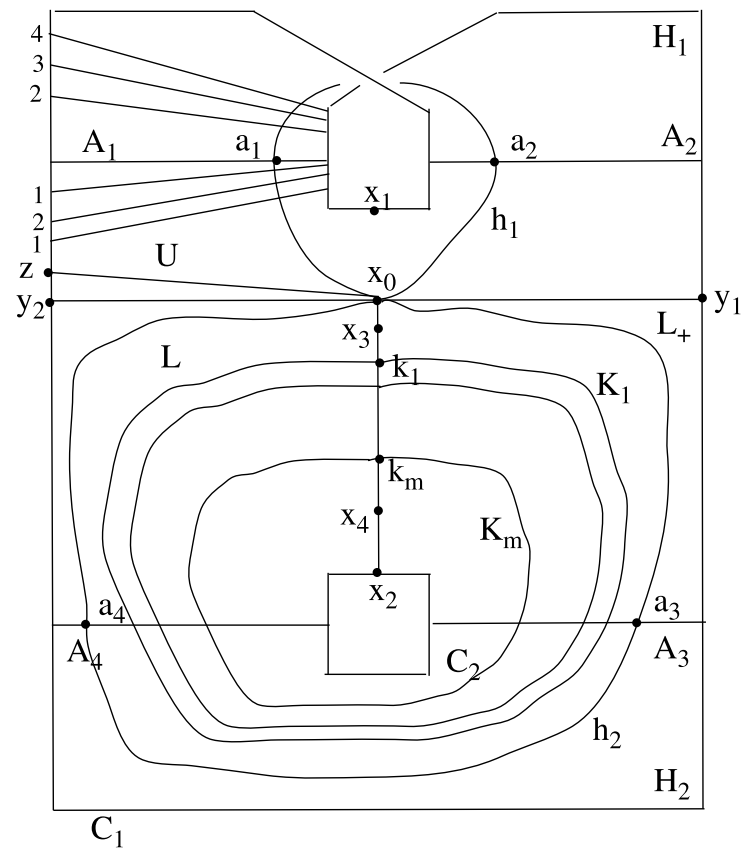

Figure 6

all the fixed points of $g$ in $U$ are therefore in $C_{1}$, we conclude that $g$ has no fixed points except those on $\partial X$ which are the fixed points of $f$ on $\partial X$. This completes the definition of $g$ on $X$ and, since it has no fixed points in the interior of $X$, we have demonstrated that $N_{\partial}(f)=M F_{\partial}[f]$.

Theorem 10 If $f:(X, \partial X) \rightarrow(X, \partial X)$ is a boundary semi-essential map such that $\operatorname{Im}_{\partial}(f)=2$, then $N_{\partial}(f)=M F_{\partial}[f]$.

Proof The proof is divided into four cases, depending on whether or not the boundary components are mapped to themselves and which of the two boundary maps is essential. The case in which each boundary component is mapped to itself by $f$, that $f_{1}$ is essential and $f_{2}$ is inessential is immediate from Lemma 8. Next we consider the case where still $f_{1}$ is essential and $f_{2}$ is inessential but now $f\left(C_{1}\right)=C_{2}$ and $f_{2}\left(C_{2}\right)=x_{1}$. Since $f_{2}$ is inessential, the components of $f^{-1}(A)$ are still the ones pictured in Figure 6 . However, the arcs of type $[1,3]$ would now be labelled $3434 \ldots$ because only $A_{3}$ and $A_{4}$ intersect $C_{2}$. Clearly, both endpoints of each arc of type $[1,3]$ must map to the same point. Thus we can define a map $g$ such that $g(U) \subseteq C_{2}$ and $g\left(\left[y_{1}, y_{2}\right]\right)=x_{2}$. Our definition of $g$ on $L$ will again consist of defining it on $\left[x_{0}, x_{2}\right]$ and extending to 
all of $L$ by annulus extensions. First, $g$ maps $\left[x_{0}, x_{3}\right]$ homeomorphically onto $\left[x_{2}, x_{0}\right]$ with $g\left(x_{3}\right)=x_{0}$, then $g$ will have exactly one fixed point on $\left[x_{0}, x_{3}\right]$. Next $g$ sends $\left[x_{3}, k_{1}\right]$ to the arc in $h$ that connects $x_{0}$ to $f\left(k_{1}\right)$. As before, we continue in this manner so that $g$ maps $\left[k_{1}, x_{4}\right]$ to $h$. Finally, $\left[x_{4}, x_{2}\right]$ is mapped homeomorphically onto the line segment $\left[x_{0}, x_{1}\right]$. Extending $g$ to all of $L$ by annulus extensions, we note that the map $g$ has only a single fixed point on all of $X$. Since the Lefschetz number of $f$ is nonzero, we conclude that $N_{\partial}(f)=M F_{\partial}[f]=1$ in this case.

The remaining cases are (1) $f_{1}$ is an inessential map of $C_{1}$ to itself and $f_{2}$ is an essential map on $C_{2}$ and (2) $f_{1}$ is an inessential map from $C_{1}$ to $C_{2}$ and $f_{2}$ is an essential map from $C_{2}$ to $C_{1}$. Both cases follow by Lemma 6 from cases we have already established.

The two results that follow complete the proof of Theorem 2 by analyzing the cases in which $f$ is boundary semi-essential with $\operatorname{Im}_{\partial}(f)=1$.

Theorem 11 If $f:(X, \partial X) \rightarrow(X, \partial X)$ is a boundary semi-essential map such that $f(\partial X)=C_{1}$ and $f_{1}$ is essential or if $f(\partial X)=C_{2}$ and $f_{2}$ is essential, then $N_{\partial}(f)=M F_{\partial}[f]$.

Proof The case in which $f(\partial X)=C_{1}$ and $f_{1}$ is essential is included in Lemma 8 and the other case then follows by Lemma 6 .

Theorem 12 If $f:(X, \partial X) \rightarrow(X, \partial X)$ is a boundary semi-essential map such that $f(\partial X)=C_{2}$ and $f_{1}$ is essential or if $f(\partial X)=C_{1}$ and $f_{2}$ is essential, then $M F_{\partial}[f]-N_{\partial}(f) \leq 1$.

Proof We will consider only the case that $f(\partial X)=C_{2}$ and $f_{1}$ is essential since the other case will follow from it by Lemma 6 . In contrast to the two previous theorems, we cannot apply Lemma 8. However, the fact that $f_{1}$ is essential still allows us to use Theorem 6 to reduce the possible types of arcs among the components of $f^{-1}(A)$ to the four types listed there. Moreover, since $f_{2}$ is inessential, we may assume that no point of $C_{2}$ is mapped to $A$ and therefore the only type of arc is $[1,3]$. Theorem 4 thus assures us that we may still use Figure 6 for the present case. We will construct a map $g$ that is boundary-preserving homotopic to $f$ with the same number of fixed points on $\partial X$, that is $N(\bar{f})$ of them, and one fixed point in the interior of $X$. The construction is a modification of the proof of Lemma 8, as follows. We may homotope $f$ on $C_{1}$ without changing the fixed points but mapping all of $C_{1} \cap L$ 
to $x_{2}$. Define $g:\left[x_{0}, x_{2}\right] \rightarrow\left[x_{0}, x_{2}\right] \cup h$ by sending $\left[x_{0}, x_{3}\right]$ to $x_{0}$ and defining $g$ on $\left[x_{3}, x_{2}\right]$ exactly as in the proof of Lemma 8. Extending $g$ to $L$ by annulus extensions gives us a map without any fixed points on the interior other than $x_{0}$.

In order to define $g$ on $U$, observe that both endpoints of a component of $f^{-1}(A)$ in $U$ are mapped to the same point of $C_{2} \cap A$. Let $V$ be the component of $U-f^{-1}(A)$ containing $\left[y_{1}, y_{2}\right]$, then $g$ can map $U-V$ to $C_{2}$ in the same way it mapped $U$ to $C_{1}$ in the proof of Lemma 8. The image under $g$ of the boundary of $V$ is contained in $\left[x_{0}, x_{2}\right] \cup T_{2}$. Since this set is contractible, we may extend $g$ from $\partial V$ to $V$ and thus $g$ is defined on all of $X$. There are no fixed points of $g$ in $U$ except $x_{0}$ and those on $C_{1}$. We conclude that $g$ has a single fixed point on the interior of $X$.

We conclude this paper with an observation about the difference between $M F_{\partial}[f]$ and $N_{\partial}(f)$ for $f$ a boundary-preserving map of the punctured Möbius band $X$. An example in [11] shows that these are not always equal. In this paper we studied the question of equality by considering a classification of boundary-preserving maps based on their restrictions to the two boundary components of $X$. From Theorems $7-12$ we can see that the only kind of map $f:(X, \partial X) \rightarrow(X, \partial X)$ for which $N_{\partial}(f)$ does not equal $M F_{\partial}[f]$ is one like the example from [11, which shows that Theorem 12 cannot be improved.

\section{References}

[1] Brouwer, L.: Über die Minimalzahl der Fixpunkte bei den Klassen von eindeutigen stetigen Transformationen der Ringfläschen, Math. Ann. 82, 94-96 (1921).

[2] Brown, R., Sanderson B.: Fixed points of boundary-preserving maps of surfaces, Pacific J. Math. 158, 243-264 (1993).

[3] Buoncristiano, S., Rourke, C., Sanderson, B.: A Geometric Approach to Homology Theory, Cambridge Univ. Press, 1976.

[4] Heath, P.: A Nielsen type number for fibre preserving maps, Top. Appl. 53, 19-35 (1993).

[5] Heath. P., Keppelmann, E., Wong, P.: Addition formulae for Nielsen numbers and the Nielsen type numbers of fiber preserving maps, Top. Appl. 67, 133-157 (1995).

[6] Jiang, B.:, Commutativity and Wecken properties for fixed points on surfaces and 3-manifolds, Top. Appl. 53, 221-228 (1993). 
[7] Jiang, B., Guo, J.: Fixed points of surface diffeomorphisms Pacific J. Math. 160, 67-89 (1993).

[8] Jiang B.: The Wecken property of the projective plane, in 'Nielsen Theory and Reidemeister Torsion', Banach Center Publications 49, 223-225 (1999).

[9] Kelly, M.: Minimizing the number of fixed points for self-maps of compact surfaces, Pacific J. Math. 126, 81-123 (1987).

[10] Kelly, M.: The relative Nielsen number and boundary-preserving surface maps, Pacific J. Math. 161, 139-153 (1993).

[11] Kelly, M.: Fixed points of boundary-preserving maps on punctured projective planes, Top. Appl. 124, 145-157 (2002).

[12] Nolan, J.: Fixed points of boundary-preserving maps of punctured discs, Top. Appl. 73, 57-84 (1996).

[13] Schirmer, H.: A relative Nielsen number, Pacific J. Math. 122, 459-472 (1986).

[14] Skora, R.: The degree of a map between surfaces, Math. Ann. 276, 415-423 (1987).

[15] Wecken, F.: Fixpunktklassen, III, Math. Ann. 118, 544-577 (1942).

Department of Mathematics, University of California

Los Angeles, CA 90095-1555, USA

and

Department of Mathematics and Computer Science, Loyola University

6363 St. Charles Avenue, New Orleans, LA 70118, USA

Email: rfb@math.ucla.edu and kelly@loyno.edu

Received: 21 November 2002 Revised: 15 October 2003 